\title{
Stable Membraneless Complex Coacervate Microdroplets
}

\author{
Shang Gao, ${ }^{1,+}$ Samanvaya Srivastava, ${ }^{1,2,3,4, *}$ \\ ${ }^{1}$ Department of Chemical and Biomolecular Engineering, University of California, Los Angeles, Los Angeles, CA 90095, USA. \\ ${ }^{2}$ California NanoSystems Institute, University of California, Los Angeles, Los Angeles, CA 90095. \\ ${ }^{3}$ Center for Biological Physics, University of California, Los Angeles, Los Angeles, CA 90095. \\ ${ }^{4}$ Institute for Carbon Management, University of California, Los Angeles, Los Angeles, CA 90095.
}

\begin{abstract}
Complex coacervate microdroplets are membraneless compartments that selectively sequester biological molecules from their surroundings and enhance bioreactions. Yet, their use as protocell models and bioreactors has been limited owing to a lack of feasible strategies to prevent their uncontrolled coalescence. Herein, we introduce an approach to mitigate coalescence of complex coacervate microdroplets using comb polyelectrolytes as stabilizers while retaining the membraneless coacervate-water interfaces, creating membraneless coacervate emulsions with months-long stabilities. Tunability of microdroplet size and stability is achieved by regulation of comb polyelectrolyte concentration and molecular weight. Importantly, the comb polyelectrolyte-stabilized coacervate microdroplets spontaneously sequester and retain proteins over extended periods. Moreover, enhanced catalytic activity of proteins and significant (up to 10-fold) acceleration of bioreactions is achieved in stabilized complex coacervate dispersions, even when stored for up to 48 hours. Our findings are expected to expand the utility of complex coacervate microdroplets as artificial protocells, encapsulants, and bioreactors, and also facilitate their use in pharmaceutical, agricultural, food, and cosmetics formulations.
\end{abstract}

\section{INTRODUCTION}

Stabilization of complex coacervate microdroplets against coalescence is the primary challenge limiting their utility in bottom-up synthetic biology and biotechnology. ${ }^{1-5}$ These membraneless microdroplets form owing to liquid-liquid phase separation in aqueous media and possess numerous attributes desired in lipid-free protocell models ${ }^{6-8}$ and colloidal bioreactors, ${ }^{9,10}$ including spontaneous self-assembly leading to compartmentalization, stability across a wide range of physiochemical conditions (temperature, $\mathrm{pH}$, and ionic strength $)^{11-13}$, and crowded environments that mimic the interior of cells. ${ }^{2,14-16}$ Moreover, the self-assembly processes that drive coacervation result in highly selective sequestration of (bio)molecules into the crowded coacervate environments. ${ }^{1,2,15-18}$ Concomitantly, the membraneless coacervate-water interface facilitates rapid transport of small molecules, resulting in significant acceleration of bioreactions in coacervate microdroplets. ${ }^{1,2,15,16,19-24}$ However, the membraneless coacervate-water interface that facilitates many of the bio(techno)logical functions of the coacervate microdroplets also facilitates their coalescence, resulting in their rapid coarsening. ${ }^{24}$ Synthetic complex coacervate dispersions typically condense into aqueous polymer-rich and polymer-lean macro-phases within minutes to hours of mixing, ${ }^{11,25}$ reducing the interfacial area between the coacervates and their surroundings and restricting their functionality and utility. Thus, deployment of complex coacervates as protocell models, ${ }^{1-5}$ protein encapsulants ${ }^{26}$ and self-assembled bioreactors ${ }^{9,10}$ have been limited to low concentration ${ }^{2,27}$ or immobilized $^{9,10,28}$ dispersions where droplet coalescence remains restricted.

Recent attempts to stabilize complex coacervates have been inspired by strategies adopted to stabilize hydrophobic phases (oils, lipids, etc.) in water. Conjugation of polyelectrolytes with a neutral block has been employed to restrict the coarsening of the coacervates at the nanoscale, resulting in complex coacervate core micelles. ${ }^{29-32}$ While these micelles have been successfully employed as delivery vehicles for charged biomacromolecules (nucleic acids and proteins), ${ }^{32-34}$ their small size and relatively low loading capacity for biomolecules have limited their use as viable protocell models and bioreactors. Efforts to stabilize coacervate microdroplets have primarily relied on introduction of additional membranous interfaces or interfacially adsorbed colloids around the droplets. For instance, fatty acids, ${ }^{3,35}$ amphiphilic proteins, ${ }^{21,23,36}$ and terpolymers, ${ }^{16,37-41}$ lipid bilayers, ${ }^{42-45}$ and lipid vesicles ${ }^{46}$ have been incorporated in the complex coacervate dispersions to assemble at the coacervate-water interface and provide steric stabilization to the coacervate microdroplets against coalescence. However, these approaches achieve stabilization at the expense of introducing a hydrophobic region around the droplets that limit the transport of small hydrophilic molecules across them as well as the capability of the microdroplets to sequester biomolecules from their surrounding solutions, require cumbersome preparation steps, and are markedly sensitive to the mixing protocols. Hitherto, viable strategies for stabilization of membraneless complex coacervate droplets have not been demonstrated.

Herein, we introduce a new paradigm for stabilization of complex coacervate microdroplets while retaining the membraneless coacervate-water interfaces as well as their unique features that distinguish them from membrane-stabilized vesicular protocell models. Our approach relies on employing comb polyelectrolytes as stabilizers that are posited to introduce steric repulsions among the microdroplets and impart long-term stability to the microdroplet dispersions. In effect, we create membraneless complex coacervate emulsions. We highlight the simplicity, the versatility, and the robustness of the stabilization approach and illustrate the tunability of microdroplet size and stability by varying the comb polyelectrolyte characteristics and 
concentrations. Moreover, we demonstrate that the comb polyelectrolyte-induced stabilization approach does not interfere with spontaneous sequestration of proteins into the coacervate microdroplets. A coupling of macromolecular crowding in the coacervate environment and unhindered transport of small molecule facilitated by large area of the stabilized microdroplet interfaces is shown to enhance the catalytic activity of proteins substantially, and more importantly, retain the enhanced activity over extended time periods. Taken together, our stabilization approach for complex coacervate microdroplets and the advantageous attributes of their stabilized dispersions promise to usher the next generation of complex coacervatebased protocells, encapsulants, stabilizers, and bioreactors.

\section{MATERIALS AND METHODS}

Materials. Poly(acrylic acid sodium salt) (PAA, molecular weight $5100 \mathrm{~g} / \mathrm{mol}$, in powder form) was purchased from Millipore Sigma. Poly(diallyldimethylammonium chloride) (PDADMAC) (molecular weight $8500 \mathrm{~g} / \mathrm{mol}$ ) was purchased from Polysciences, Inc. as a $28 \mathrm{wt} \%$ aqueous solution. Comb polyelectrolytes (MasterGlenium7500, MVA2808, MVA2500 and MVA2453) were supplied by BASF. Bovine serum albumin (BSA, molecular weight $66 \mathrm{kDa}$, lyophilized powder), lipase from Candida rugosa (molecular weight $58 \mathrm{kDa}$, lyophilized powder), and $p$-nitrophenyl butyrate was purchased from Millipore Sigma. Fluorescein isothiocyanate-labelled bovine serum albumin (FITC-BSA) was synthesized ${ }^{47}$ using fluorescein isothiocyanate (FITC, purchased from Millipore Sigma). A 10 $\mathrm{mg} / \mathrm{mL}$ BSA solution was prepared with $50 \mathrm{mM}$ sodium bicarbonate buffer solution at $\mathrm{pH}=8.5 .50 \mathrm{uL} 1 \mathrm{mg} / \mathrm{mL}$ fluorescein isothiocyanate solution in DMSO was slowly added into the BSA solution in dark followed by a 2-hour reaction. Purification by dialysis was carried out to remove free fluorescein isothiocyanate. The labeled FITC-BSA was stored in refrigerator before use.

Preparation of Complex Coacervate Dispersions. Stock solutions of the linear polyelectrolytes, the comb polyelectrolyte and water were mixed in appropriate amounts to produce complex coacervate dispersions of $50 \mu \mathrm{L}$ for microimaging, $250 \mu \mathrm{L}$ for DLS measurements, or $2 \mathrm{~mL}$ for macroscopic observations. All dispersions contained charge-matched amounts of the oppositely charged linear polyelectrolytes. In dispersions containing proteins (FITC-BSA or lipase), protein stock solutions were also added to the dispersions following the adding sequence: PDADMAC, water, comb polyelectrolyte, protein, and PAA.

Optical Microscopy. Inverted Confocal microscope (Leica, Confocal SP8-STED/FLIM/FCS) was employed to image all complex coacervate dispersions (in brightfield camera settings). A small aliquot $(\sim 6 \mu \mathrm{L})$ of the coacervate mixtures (at different times after mixing) was sandwiched between silanized glass cover slips. Coacervate droplets were imaged using a 100-X oil-immersion objective.

Fluorescence Microscopy. Inverted confocal microscope (Leica, Confocal SP8-STED/FLIM/FCS) was employed to image all complex coacervate dispersions in fluorescence settings, with the excitation and emission spectrum wavelengths in the pre-set FITC Leica settings. A small aliquot $(\sim 6 \mu \mathrm{L})$ of the coacervate mixtures (at different times after mixing) was sandwiched between silanized glass cover slips. Coacervate droplets were imaged using a 100-X oil-immersion objective.

Turbidity Measurements. Turbidity measurements of all dispersions were carried out using a plate reader equipped with a UV spectrophotometer (Tecan Infinite M1000 Pro). $250 \mu \mathrm{L}$ dispersions were prepared in a 96-well plate (flat bottom, transparent, Costar). The well-plate was shaken for 10 seconds before turbidity measurements. Turbidity scale was defined by $100 \%-T_{580 \mathrm{~nm}} \%=$ $10^{(2-A b s o r b a n c e)}$.

Absorbance Measurements. Absorbance at $405 \mathrm{~nm}$ of the coacervate samples were measured using UV spectrophotometer (Tecan Infinite M1000 Pro). $50 \mu \mathrm{L}$ of samples (complex coacervates or controls) were placed in the 96 -well plate. $150 \mu \mathrm{L}$ of $p$-nitrophenyl butyrate solutions, prepared in $\mathrm{pH}=7$ phosphate buffered saline (PBS), were added into each well (yielding $0.1 \mathrm{mM} p$-nitrophenyl butyrate in each well) before measuring the absorbance at $405 \mathrm{~nm}$ for $60 \mathrm{mins}$ at $40 \mathrm{~s}$ intervals. The $p$-nitrophenyl butyrate solution was prepared by dissolving $5 \mu \mathrm{L} p$-nitrophenyl butyrate in in $5.3 \mathrm{~mL}$ isopropanol, followed by addition of $500 \mu \mathrm{L}$ of this solution to $20 \mathrm{~mL}$ $\mathrm{pH}=7$ PBS.

Dynamic Light Scattering. Dynamic light scattering (DLS) analysis was carried out on Malvern Zetasizer Nano to assess the hydrodynamic radius of the microdroplets in all the dispersions. Three samples of each dispersion were prepared, and three trials were carried out on each sample. Standard deviations of the particle sizes in the dispersions were calculated on the triplicate samples and three measurements of each sample.

\section{RESULTS}

Comb Polyelectrolytes Stabilize Complex Coacervate Microdroplets

Figure 1 presents a visual comparison between unstable and stable dispersions of model complex coacervates. Both the complex coacervate dispersions comprise a charge-matched mixture of oppositely charged polyelectrolytes, poly(acrylic acid sodium salt) (PAA, molecular weight $M W=5100 \mathrm{~g} / \mathrm{mol})$ and poly(diallydimethylammonium chloride) (PDADMAC, $M W=8500 \mathrm{~g} / \mathrm{mol}$ ). The stabilized dispersion also contained a small amount of polyacrylic acid-comb-polyethylene glycol (PAA-c-PEG, $M W=39467 \mathrm{~g} / \mathrm{mol}$, $26 e^{-}$at $\mathrm{pH}=6$, PEG $M W=3000 \mathrm{~g} / \mathrm{mol}$ ) comb polyelectrolytes. Both dispersions were turbid upon initial mixing of their constituents (Figure 1A and 1B), owing to the presence of complex coacervate microdroplets. Rapid coalescence of the microdroplets and gravity-induced sedimentation in the unstable dispersions resulted in clear solutions with distinct complex coacervate and supernatant phases within a few hours (Figure 1A). In contrast, stabilized coacervate microdroplets remained dispersed up to 48 hours (Figure 1B), with turbidity levels staying nearly constant (Figure 1C). Micrographs of the as-mixed unstable and stable dispersions both show spherical complex coacervate microdroplets, as expected upon spontaneous mixing of the constituents (Figures $1 \mathrm{~A}$ and $1 \mathrm{~B}$, respectively). However, while the unstable droplets coalesced into a homogenous phase, spherical microdroplets were still clearly visible in the stable dispersions after 48 hours.

Comb polyelectrolytes imparted exceptional stability to the coacervate microdroplets. Over 4 months, the coacervate droplets sediment, yielding clear solutions (Figure 1B). Yet, the microdroplets did not coalesce and maintained their spherical morphology, as is evident from micrographs of the dense dispersion of coacervate microdroplets, highlighting the effectiveness of comb polyelectrolytes in preventing microdroplets coalescence (Figure 1B). Moreover, the sedimented droplets readily dispersed upon gentle shaking of the vials (Figure 1B). Correspondingly, turbidity in the shaken vials recovered to levels similar to the as-mixed emulsions and persisted for a few hours after shaking (Figure 1C). 

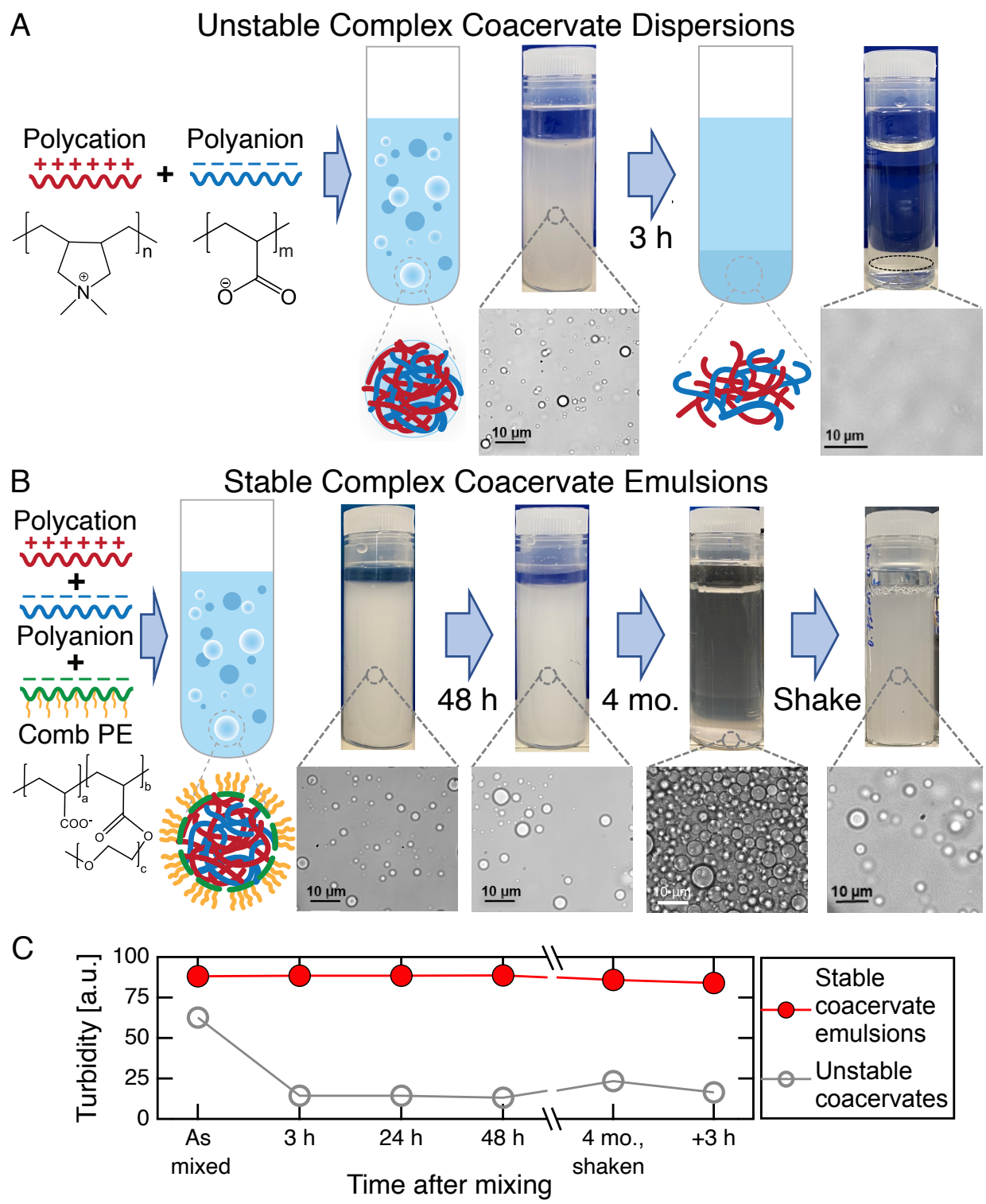

Figure 1. Comb polyelectrolytes stabilize complex coacervate microdroplets. (A) Aqueous mixture of oppositely charged polyelectrolytes (38.8 mM ionizable groups, 1:1 charge ratio) form turbid dispersions of complex coacervate droplets. The coacervate droplets coalesce readily (within 3 hours) to form a homogenous, transparent coacervate phase. The black dashed line denotes the location of the interface between the polymer dense coacervate phase (at the bottom) and the polymer-lean supernatant phase (at the top). Corresponding micrographs depict coacervate droplets in the as mixed dispersions that coalesce into a homogenous coacervate phase within 3 hours. (B) Stable complex coacervate emulsions obtained by preparing the same solutions as in (A) with an additional component - anionic comb polyelectrolytes (4.8 mM ionizable groups, see Table S1). The stable complex coacervate emulsions are turbid when prepared and remained turbid for up to 48 hours. Corresponding micrographs reveal distinct micron-sized droplets in the emulsions. Over 4 months, the coacervate microdroplets settle to the bottom of the vial but do not coalesce, leading to a dense emulsion at the bottom of the vial as revealed from the micrograph of the emulsions taken from the bottom of the vials. Gentle shaking of the vial led to re-dispersion of the coacervate microdroplets, resulting in turbid emulsions. In (A) and (B), scale bars: $10 \mu \mathrm{m}$. (C) Turbidity is a key indicator of the stability of the emulsions. Turbidity in unstable coacervate dispersions decreased readily within 3 hours, resulting in clear solutions. In contrast, turbidity in the stable coacervate dispersions did not decay up to 48 hours. 4-month aged dispersions exhibit similar levels of turbidity upon shaking as the freshly prepared samples and remained turbid for up to 3 hours after shaking.

In typical emulsions, oil-water interfaces possess a high interfacial tension and thus provide a strong driving force for interfacially active molecules to self-assemble at the interfaces. In contrast, coacervatewater interfaces are characterized by low interfacial tensions, ${ }^{48,49}$ thus requiring multipoint anchoring to localize the polymeric stabilizers at the interface. The unique architecture of the comb polyelectrolytes, consisting of multiple PEO chains attached to the PAA backbone, is posited to enable such multipoint anchoring of the backbone on the surface of the coacervate droplets while the neutral side chains remain in the supernatant and restrict the complete assimilation of the backbone in the coacervate phase. The PEO side chains, with a Flory radius of $\sim 5 \mathrm{~nm}$, are thus hypothesized to form a dense corona around the microdroplets, providing steric repulsion among them and restricting their coalescence, thus imparting long- 
term stability to the dispersions.

Reducing the strength of the steric repulsion between the coacervate microdroplets can therefore be anticipated to hinder their stabilization. This was consistent with our observations that while comb polyelectrolytes with similar backbone charge density but varying lengths of the PEG side chains were all effective at inhibiting droplet coalescence (Figures S1 and S2, Table S1), decreasing the PEG side chain molecular weight led to poorer stabilization of the coacervate droplets and their faster settling (Figure S2). Similarly, diblock polymers comprising a charge-bearing block and a neutral block do not impart stability to coacervate droplets, ascribable to their insufficient interfacial absorption resulting in their assimilation of the polymers in the coacervate droplets; our representative experiments led to formation of dense complexes (Figure S3). At the same time, the sterically hindered inability of the comb polyelectrolytes to assimilate into the coacervate phase also meant that, when mixed with linear polyelectrolytes, comb polyelectrolytes did not form any self-assembled structures (Figure S3).
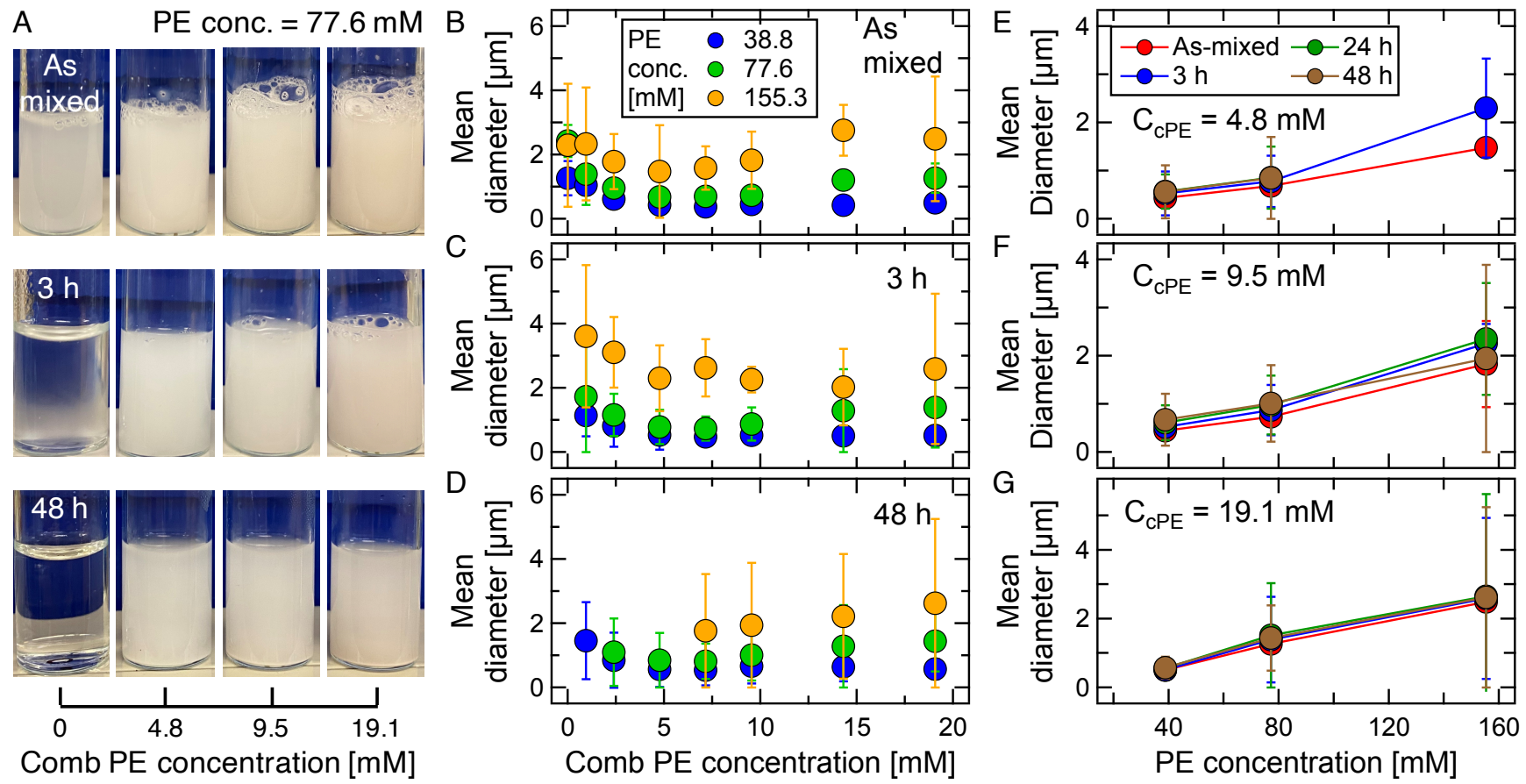

Figure 2. Droplet size in stable complex coacervate dispersions is controlled by the linear polyelectrolyte and the comb polyelectrolyte concentrations. (A) Stable complex coacervate dispersions comprising oppositely charged polyelectrolytes (155.3 mM ionizable groups, $1: 1$ charge ratio) with increasing concentration, expressed in $\mathrm{mM}$ of ionizable groups, of comb polyelectrolyte stabilizers. Photographs are taken from different times, indicated as "as mixed", 3 hours, and 48 hours after mixing. Turbidity in the samples is a key factor for indicating the stability of the dispersions. (B-D) Evolution of the mean diameter (by volume) of the complex coacervate droplets with comb polyelectrolyte concentration (expressed in $\mathrm{mM}$ of ionizable groups) in dispersions comprising polyelectrolyte concentrations (expressed in concentration of ionizable groups, 1:1 charge ratio) ranging from $38.8 \mathrm{mM}$ to $155.3 \mathrm{mM}$ measured at different times, indicated as "as mixed” (B), 3 hours (C), and 48 hours (D) after mixing. Droplet size underwent a nonmonotonic trend - decreasing initially before increasing and finally plateauing with comb polyelectrolyte concentration. (E-G) Evolution of the mean diameter (by volume) of the complex coacervate droplets with increasing polyelectrolyte concentrations (expressed in concentration of ionizable groups, 1:1 charge ratio) and time after mixing, at constant comb polyelectrolyte concentration (expressed in $\mathrm{mM}$ of ionizable groups) of $4.8 \mathrm{mM}(\mathrm{E}), 9.5 \mathrm{mM}(\mathrm{F})$, and $19.1 \mathrm{mM}$ (G). Droplet sizes increase almost linearly with PE concentration. Temporal coarsening of droplets reduced with increasing comb polyelectrolyte concentrations. In (D) and (E), settling occurred within 24 hours for samples with the highest polyelectrolyte concentration and low comb polyelectrolyte concentrations, and therefore droplet sizes are not shown. In (B-G), droplet sizes were measured using dynamic light scattering and averaged from triplicate samples at each concentration and three measurement trials for each sample. Filled symbols denote data measured from unperturbed samples. Errors are calculated through the mean standard deviations of the measurements. Error bars are shown when they are larger than the symbols. The size distribution of the coacervate droplets was obtained by dynamic light scattering (DLS) operated in backscattering mode since optical microscopy depicts only the large droplets that sediment to the bottom of the vials.

Relative Concentrations of Linear and Comb Polyelectrolytes Dictate Complex Coacervate Microdroplets Size and Stability

Both the extent of stabilization and the size of the stabilized complex coacervate droplets is controllable by tuning the relative concentrations of the comb and the linear polyelectrolytes. As shown in Figure $2 \mathrm{~A}$, increasing the comb polyelectrolyte concentration, $C_{C P E}$ in dispersions containing a constant concentration of the constituent polyelectrolytes (PAA and PDADMAC) $C_{P E}$ resulted in reduced settling of the complex coacervate droplets. Concomitantly, the mean diameter of the droplets $D$, measured using dynamic light scattering, in as-mixed dispersions containing a constant $C_{P E}$ decreased initially before increasing marginally and plateauing with increasing $C_{C P E}$ (Figure 2B - 2D). The total interfacial area that can be stabilized is expected to be proportional to $C_{C P E}$. Thus, upon increasing $C_{C P E}, D$ decreased until all droplets possessed a monolayer of comb polyelectrolytes on their surfaces. Further increasing $C_{C P E}$ 
resulted in multilayer adsorption on the droplets surfaces, resulting in slight increase of the droplets sizes, followed by saturation of the droplet surfaces, and plateauing of the droplet size. The extent of saturation of the droplet surfaces also dictated their temporal stability. While marginal coarsening of the droplets and settling was observed over 48 hours at low $C_{C P E}$ (Figure $2 \mathrm{E}$ and $2 \mathrm{~F}$ ), droplet size distribution remained nearly unchanged at high $C_{C P E}$ (Figure 2G).At the same time, the total volume of the complex coacervate phase is expected to be proportional to $C_{P E} \cdot{ }^{11,50}$ Therefore, larger $C_{C P E}$ were required to stabilize dispersions with high $C_{P E}$. Correspondingly, $D$ was found to increase linearly with increasing $C_{P E}$ at a constant $C_{C P E}$ (Figure 2E-G). In dispersions with low $C_{C P E}$ and high $C_{P E}$ (Figure $2 \mathrm{E})$, the comb polyelectrolytes were insufficient to stabilize the larger volume of the coacervate phase into small droplets that remain suspended in the emulsions, resulting in unstable dispersions.

Dilution of the stabilized dispersions did not undermine the stability of the complex coacervate microdroplets. Moreover, dilution from concentrated homogenous mixtures of the linear and comb polyelectrolytes resulted simultaneous coacervation of the linear polyelectrolytes and interfacial absorption of the comb polyelectrolytes. Thus, a single-phase mixture of the oppositely charged linear polyelectrolytes and the comb polyelectrolytes, when diluted 12-, 24-, 30-, 48-, and 120-fold by addition of water, resulted in stable complex coacervate emulsions that remained turbid up to 48 hours after dilution (Figure S4).

Order of mixing of the linear and comb polyelectrolytes also did not affect the stability of the microdroplets. The results shown in in Figures 1 and 2 followed a mixing order wherein the comb polyelectrolyte was introduced in the mixture after the polycation but before the polyanion. Other mixing sequences, including mixing of the polycation and the polyanion before the addition of the comb polyelectrolyte and introduction of the comb polyelectrolyte first followed by the addition of the polycation and the polyanion also led to stable dispersions of the complex coacervate droplets (see Figure S5). These results, when combined with resilience against dilution, demonstrate the strong interfacial affinity of the comb polyelectrolytes in aqueous complex coacervate dispersions and highlighting the versatility of the approach demonstrated here to produce stable coacervate emulsions.

Proteins Sequester Spontaneously in Stabilized Complex Coacervate Microdroplets

Proteins sequester spontaneously into the comb polyelectrolytestabilized complex coacervate microdroplets. Mixing of fluorescein isothiocyanate labeled bovine serum albumin (FITC-BSA) with the linear and comb polyelectrolytes resulted in strong localization of the proteins inside the stabilized droplets, as monitored by fluorescence imaging. BSA, with a net negative charge of $18 e^{-}$at $\mathrm{pH} \sim 6$, was found to distribute uniformly inside the droplets, as indicated by the overlaid brightfield and fluorescent images (Figure 3A, rows 1 and 2). Moreover, the microdroplet morphology (Figure 3A, rows 1 and 2) and sizes (Figure 3B) in as-mixed dispersions were similar in unstable and stabilized dispersions. Yet, while the unstable proteinloaded coacervate droplets (Figure 3A, row 1) coalesced readily, resulting in clear solutions with distinct coacervate and supernatant phases, the stabilized coacervate microdroplets maintained their stability up to 48 hours (Figure 3A, row 3 ). The microdroplets coarsened marginally (Figure 3B), akin to coacervate microdroplets without proteins (Figure 2E-G). Moreover, FITC-BSA remained localized within the stabilized microdroplets up to 48 hours (Figure $3 \mathrm{~A}$, row 3 ).
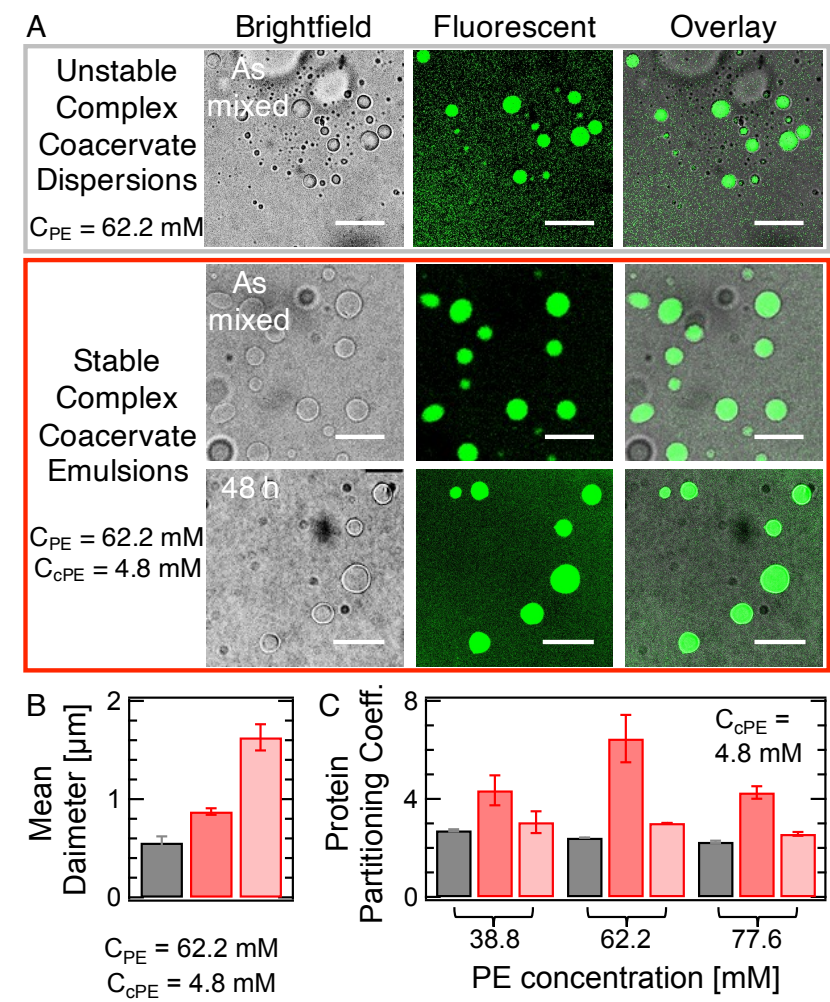

$\square$ Unstable $\square$ Stable, as mixed $\square$ Stable, $48 \mathrm{~h}$

Figure 3. Proteins partition preferentially in coacervate droplets in the stable coacervate dispersions. (A) Confocal brightfield (left), fluorescence (middle) and overlayed (right) micrographs show FITC-labelled BSA localized in as mixed dispersions of unstable coacervates droplets (row 1 ) and stabilized complex coacervate microdroplets (row 2), as well as in stabilized complex coacervate microdroplets incubated for 48 hours (row 3 ). The droplets were visualized upon settling on nonadherent substrates. FTIC-BSA concentration was $1 \mathrm{mM}$ (charge concentration). Scale bars: $10 \mu \mathrm{m}$. (B) Mean diameter of FITC-BSA loaded unstable and stable coacervate microdroplets in dispersions corresponding to those depicted in (A). Mean diameters were estimated from measurements on triplicate samples and three measurement trials for each sample. (C) The protein partitioning coefficient for FITC-BSA into the coacervate phase, as estimated by the ratio of the fluorescence intensity inside and outside the droplets, for as mixed unstable dispersions, as mixed stable dispersions, and stable dispersions aged 48 hours of coacervate microdroplets as a function of polyelectrolyte concentration.

The extent of protein localization inside the microdroplets was estimated as the ratio of the fluorescent intensity inside and outside the droplets. A strong partitioning of FITC-BSA inside the stabilized coacervate microdroplets was revealed (Figure $3 \mathrm{C}$ ), in agreement with previous studies. ${ }^{15,17}$ Nominal loss of protein from the stabilized microdroplets was observed as the droplets equilibrated; the apparent protein partitioning coefficient in the stable microdroplets after 48 hours of preparation were comparable with freshly prepared unstable complex coacervate dispersions (Figure 3C). Thus, it can be surmised that comb polyelectrolyte stabilized coacervate microdroplets can be employed as aqueous protein encapsulants with a substantial protein sequestration and retention. 
A

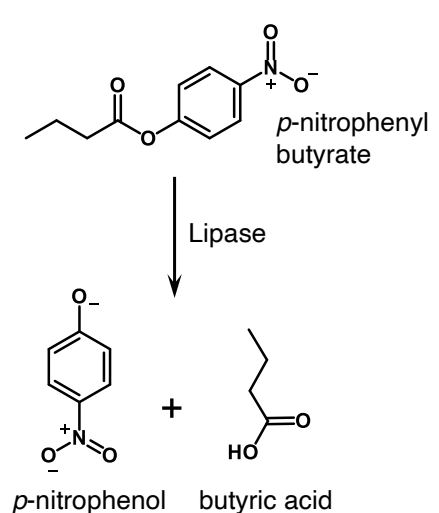

B

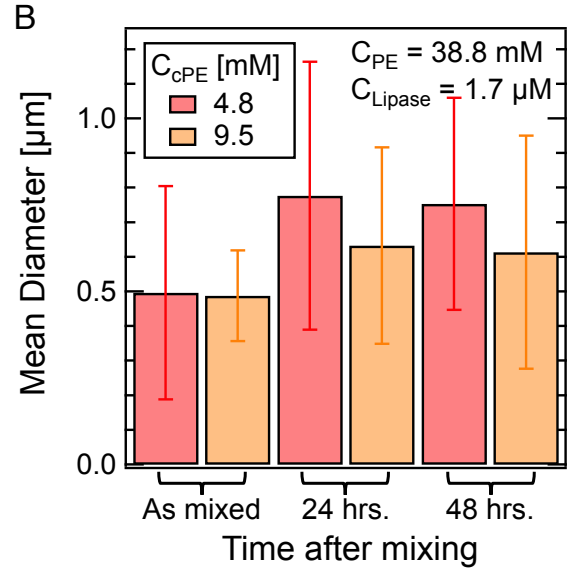

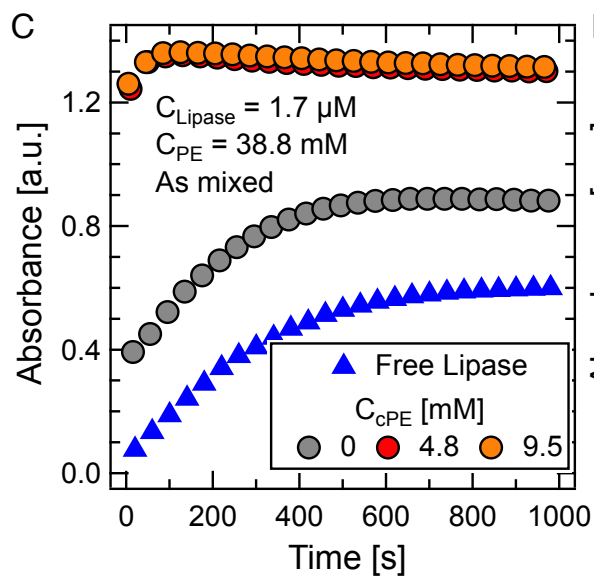
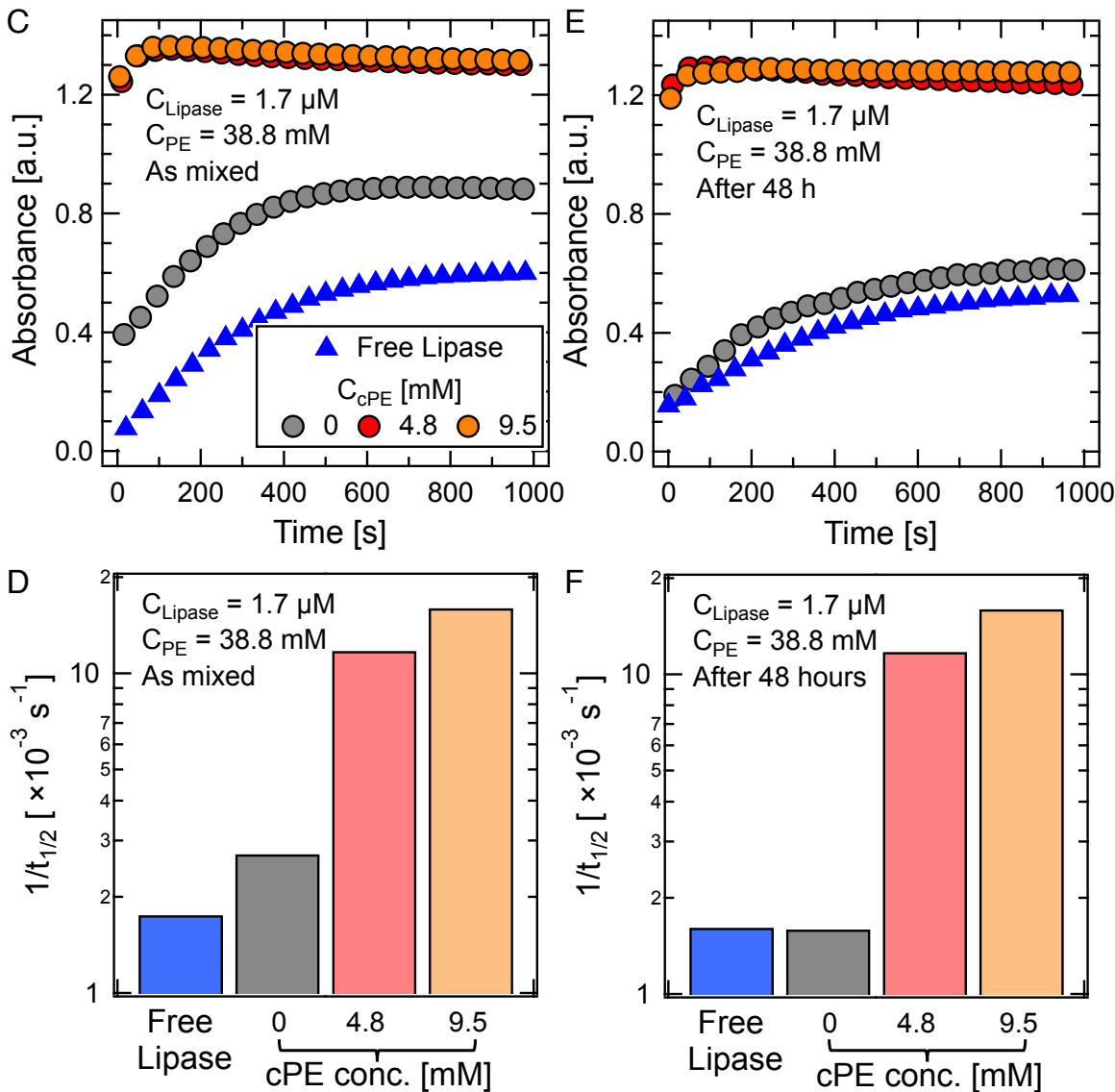

Figure 4. Activity of enzymes is markedly enhanced upon localization in stable complex coacervate microdroplets. (A) Reaction scheme depicting the lipase-mediated degradation of $p$-nitrophenyl butyrate to produce $p$-nitrophenol. (B) Temporal evolution of the mean diameter of stabilized coacervate microdroplets with co-localized lipase. Mean diameters were estimated from measurements on triplicate samples and three measurement trials for each sample. (C) Temporal evolution of the absorbance at $405 \mathrm{~nm}^{-1}$ upon addition of $p$-nitrophenyl butyrate to an aqueous solution of lipase, unstable complex coacervate dispersions with lipase localized in the unstable coacervate microdroplets, and complex coacervate dispersions where lipase is localized in stabilized microdroplets. The activity of lipase is revealed by the temporal evolution of the absorbance spectra of the $p$-nitrophenol produced upon enzymatic degradation of $p$-nitrophenyl butyrate. The absorbance spectra for coacervate dispersions evolved at a significantly faster rate as compared to lipase solution or unstable coacervate dispersions, denoting enhanced reaction rates in solutions where enzymes were localized in stabilized microdroplets. $(\mathbf{D})$ The reaction rate $\left(1 / t_{1 / 2}\right)$, estimated from the absorbance spectra, in dispersions corresponding to (C). (E and F) Temporal evolution of the absorbance at $405 \mathrm{~nm}^{-1}$ and the reaction rate $\left(1 / t_{1 / 2}\right)$ in dispersions corresponding to (C) but aged for 48 hours before addition of $p$-nitrophenyl butyrate.

Stabilized Complex Coacervate Microdroplets Facilitate Faster Enzymatic Reactions

Lipase mediated degradation of $p$-nitrophenyl butyrate to $p$-nitrophenol (Figure 4A) was carried out in stabilized complex coacervate microdroplets to highlight their utility as membraneless protocells and bioreactors. The evolution of the absorbance spectra of $p$-nitrophenol (at $\sim 405 \mathrm{~nm}$ ) provided a facile route to quantify its production rate and the catalytic activity of lipase (Figure 4C) ${ }^{51}$ Aqueous mixtures of lipase, oppositely charge linear polyelectrolytes and comb polyelectrolytes formed stable complex coacervate microdroplets wherein lipase, bearing $14 e^{-}$at $\mathrm{pH}=6$, co-localized with the linear polyelectrolytes. Enzymatic degradation of $p$-nitrophenyl butyrate in such dispersions occurred rapidly, manifesting as a fast growth of the absorbance spectra before plateauing (Figure 4C, see also Figure S6). Expectedly, the growth of the absorbance spectra in the stabilized dispersions was significantly faster than the corresponding spectra evolution in lipase solutions. Surprisingly, the spectra evolution in the stabilized dispersions were faster than even the unstable complex coacervate dispersions.

Going beyond the results suggesting co-localization of FITC-BSA (Figure 3) demonstrating that comb polyelectrolytes do not interfere with the co-localization of proteins in the coacervate microdroplets, these results indicate that comb polyelectrolytes that are anchored on the droplet surfaces (i) do not impede with the transport of small molecule substrate ( $p$-nitrophenyl butyrate) or product ( $p$-nitrophenyl) in and out of the droplets, respectively, (ii) do not interfere with the catalytic action of lipase, and (iii) promote the biodegradation reaction. Moreover, increasing the comb polyelectrolyte concentration led to a faster evolution of the absorbance spectra, indicating even further acceleration of the biodegradation of $p$-nitrophenyl butyrate to $p$-nitrophenol in the stabilized coacervate microdroplets.

An estimation of the enzymatic reaction rate $\left(1 / t_{1 / 2}\right)$ was obtained as the inverse of half of the time required for the plateauing of the absorbance spectra. Depicted in Figure 4D, it is evident that 
localization of lipase in complex coacervates led to a two-fold increase in the $p$-nitrophenol production rate. This enhancement is consistent with previous reports ${ }^{51}$ and can be attributed to enhanced macromolecular crowding in the coacervate environments. ${ }^{2,5,15,19,20,39,51}$ Stabilizing the coacervate droplets by addition of comb polyelectrolytes led to a further increase in $p$-nitrophenol production rates, with up to 5-fold increase and 10-fold increase as compared to reaction rates in complex coacervates and freely suspended lipase in solution, respectively (Figure 4D). The reaction rates were also tunable by varying the comb polyelectrolyte concentrations, with higher $C_{C P E}$ resulting in smaller droplets (Figure 4B) and higher reaction rates (Figure 4D). We posit that the coacervate microdroplets maintain a large membraneless interfacial area in the stabilized dispersions while the comb polyelectrolytes do not interfere with the transport of small molecules, resulting in swift transport of small molecules in and out of the coacervate droplets, facilitating the bioreaction.

Temporal stability of the coacervate-water interface had a distinct influence on the bioreaction rate in the complex coacervates. As the coacervate droplets coalesced into a homogenous coacervate phase in the unstable dispersions, the bioreaction rate declined markedly to approach reaction rates in aqueous protein solutions (Figures $4 \mathrm{E}$ and $4 \mathrm{~F}$ ), thus relinquishing all the advantages of carrying out the bioreaction in a coacervate environments. In contrast, the stabilized coacervate microdroplets remained in dispersed state up to 48 hours (with minor coarsening of droplets, see Figure 4B), enabling unmitigated transport of substrate and product molecules and preserving the $p$-nitrophenyl butyrate biodegradation rates (Figures $4 \mathrm{E}$ and $4 \mathrm{~F})$.

\section{DISCUSSION}

In summary, we have introduced a methodology to subside coalescence of complex coacervate microdroplets by employing comb polyelectrolyte stabilizers. The stabilized microdroplets retain their membraneless interface with their surroundings yet remain stable for months and do not coalesce even when placed in proximity of other microdroplets. Moreover, we have demonstrated that microdroplet stabilization is independent of the dispersion processing routes and a facile tuning of the microdroplet size and stability can be achieved by varying the comb polyelectrolyte size and concentrations.

Proteins, when introduced in the mixtures, partitioned spontaneously into the stabilized microdroplets, and remained localized in them up to 48 hours. Macromolecular crowding in the coacervate environment enhanced the catalytic activity of proteins by enhancing diffusion of substrate molecules and their binding affinity with protein. The stabilized coacervate-water interfaces further accelerated protein catalyzed reactions by up to 10 -fold by promoting rapid transport of the small molecule substrate and products in and out of the coacervate microdroplets. The stabilization of proteins in the microdroplets and of the microdroplets against coalescence also meant that protein-containing dispersions retained their ability to catalyze and accelerate biochemical reactions even days after preparation. Thus, the comb polyelectrolyte-stabilized coacervate microdroplets can be envisioned as stable protein storage platforms that retain the biomolecules partitioned into them and conserve their activity over extended durations. We envision that the long-term stability and the protein encapsulation attributes of the complex coacervate microdroplets will be key in their development as synthetic protocells and colloidal self-assembled microreactors sustaining and supporting cell-free biosynthesis. Furthermore, we anticipate that the stabilization approach demonstrated here provides a platform for developing complex coacervate emulsions as encapsulants and delivery vehicles in pharmaceutical, ${ }^{52}$ agricultural,,${ }^{53-56}$ food, ${ }^{57}$ and $\operatorname{cosmetics}^{58}$ formulations.

\section{ASSOCIATED CONTENT}

\section{Supporting Information}

Results from additional experiments, including stabilization experiments with other comb and block polyelectrolytes, and characteristics of the comb polyelectrolytes. The Supporting Information is available free of charge on the ACS Publications website.

\section{AUTHOR INFORMATION}

\section{Corresponding Author \\ *Email: samsri@ucla.edu}

\section{Present Addresses}

+ Department of Chemical Engineering, Stanford University, Stanford, CA 94305 , USA.

\section{Author Contributions}

The manuscript was written through contributions of all authors. All authors have given approval to the final version of the manuscript.

\section{Funding Sources}

The authors acknowledge the following funding support:

National Science Foundation grant DMR 2048285 (SS, SG)

UCLA Samueli School of Engineering startup funds (SS, SG)

\section{Notes}

SG and SS are inventors on provisional application \#63/187,031 filed on $05 / 11 / 2020$ held by University of California, Los Angeles that covers the use of comb polyelectrolytes as stabilizers for complex coacervate microdroplets.

\section{ACKNOWLEDGMENT}

The authors acknowledge helpful discussions Prof. Vivek Sharma from University of Illinois at Chicago, Prof. Jonathan Whitmer from University of Notre Dame, Prof. Karthik Nayani from the University of Arkansas Dr. Sumanth Jamadagni from P\&G, and Dr. Hari Katepalli from Dow Core R\&D.

\section{REFERENCES}

(1) Koga, S.; Williams, D. S.; Perriman, A. W.; Mann, S. PeptideNucleotide Microdroplets as a Step towards a MembraneFree Protocell Model. Nature Chemistry 2011, 3 (9), 720724. https://doi.org/10.1038/nchem.1110.

Sokolova, E.; Spruijt, E.; Hansen, M. M. K.; Dubuc, E.; Groen, J.; Chokkalingam, V.; Piruska, A.; Heus, H. A.; Huck, W. T. S. Enhanced Transcription Rates in Membrane-Free Protocells Formed by Coacervation of Cell Lysate. Proceedings of the National Academy of Sciences 2013, 110 (29), 11692-11697. https://doi.org/10.1073/pnas.1222321110.

Li, M.; Huang, X.; Tang, T. Y. D.; Mann, S. Synthetic Cellularity Based on Non-Lipid Micro-Compartments and Protocell Models. Current Opinion in Chemical Biology 2014, 22, 1-11. https://doi.org/10.1016/j.cbpa.2014.05.018.

Salehi-Reyhani, A.; Ces, O.; Elani, Y. Artificial Cell Mimics as Simplified Models for the Study of Cell Biology. Experimental 
Biology and Medicine 2017, 242 (13), 1309-1317. https://doi.org/10.1177/1535370217711441.

Abbas, M.; Lipiński, W. P.; Wang, J.; Spruijt, E. PeptideBased Coacervates as Biomimetic Protocells. Chemical Society Reviews 2021, 50 (6), 3690-3705. https://doi.org/10.1039/D0CS00307G.

(6) Szostak, J. W.; Bartel, D. P.; Luisi, P. L. Synthesizing Life. Nature 2001, 409 (6818), 387-390. https://doi.org/10.1038/35053176.

(7) Dzieciol, A. J.; Mann, S. Designs for Life: Protocell Models in the Laboratory. Chemical Society Reviews 2012, 41 (1), 7985. https://doi.org/10.1039/c1cs15211d.

(8) Hammer, D. A.; Kamat, N. P. Towards an Artificial Cell. FEBS Letters 2012, $586 \quad$ (18), 2882-2890. https://doi.org/10.1016/j.febslet.2012.07.044.

(9) Liu, J.; Tian, L.; Qiao, Y.; Zhou, S.; Patil, A. J.; Wang, K.; Li, M.; Mann, S. Hydrogel-Immobilized Coacervate Droplets as Modular Microreactor Assemblies. Angewandte Chemie International Edition 2020, 59 (17), 6853-6859. https://doi.org/10.1002/anie.201916481.

(10) Chen, Y.; Zhang, Y.; Li, M.; Liu, S.; Yang, X.; Wang, K.; Mann, S.; Liu, J. Self-Immobilization of Coacervate Droplets by Enzyme-Mediated Hydrogelation. Chemical Communications 2021, $57 \quad$ (44), 5438-5441. https://doi.org/10.1039/d1cc01483h.

(11) Srivastava, S.; Tirrell, M. V. Polyelectrolyte Complexation. In Advances in Chemical Physics; 2016; Vol. 161, pp 499-544. https://doi.org/10.1002/9781119290971.ch7.

(12) Sing, C. E.; Perry, S. L. Recent Progress in the Science of Complex Coacervation. Soft Matter 2020, 16 (12), 28852914. https://doi.org/10.1039/D0SM00001A.

(13) Friedowitz, S.; Lou, J.; Barker, K. P.; Will, K.; Xia, Y.; Qin, J. Looping-in Complexation and Ion Partitioning in Nonstoichiometric Polyelectrolyte Mixtures. Science Advances 2021, 7 (31), eabg8654. https://doi.org/10.1126/sciadv.abg8654.

(14) Aumiller, W. M.; Keating, C. D. Phosphorylation-Mediated RNA/Peptide Complex Coacervation as a Model for Intracellular Liquid Organelles. Nature Chemistry 2016, 8 (2), 129137. https://doi.org/10.1038/nchem.2414.

(15) McCall, P. M.; Srivastava, S.; Perry, S. L.; Kovar, D. R.; Gardel, M. L.; Tirrell, M. V. Partitioning and Enhanced Self-Assembly of Actin in Polypeptide Coacervates. Biophysical Journal 2018, $114 \quad$ (7), 1636-1645. https://doi.org/10.1016/j.bpj.2018.02.020.

(16) Altenburg, W. J.; Yewdall, N. A.; Vervoort, D. F. M.; van Stevendaal, M. H. M. E.; Mason, A. F.; van Hest, J. C. M. Programmed Spatial Organization of Biomacromolecules into Discrete, Coacervate-Based Protocells. Nature Communications 2020, 11 (1), 6282. https://doi.org/10.1038/s41467020-20124-0.

(17) Black, K. A.; Priftis, D.; Perry, S. L.; Yip, J.; Byun, W. Y.; Tirrell, M. V. Protein Encapsulation via Polypeptide Complex Coacervation. ACS Macro Letters 2014, 3 (10), 1088-1091. https://doi.org/10.1021/mz500529v.

(18) Douliez, J.-P.; Martin, N.; Gaillard, C.; Beneyton, T.; Baret, J.-C.; Mann, S.; Beven, L. Catanionic Coacervate Droplets as a Surfactant-Based Membrane-Free Protocell Model. Angewandte Chemie International Edition 2017, 56 (44), 1368913693. https://doi.org/10.1002/anie.201707139.

(19) Dora. Tang, T.-Y.; van Swaay, D.; DeMello, A.; Ross Anderson, J. L.; Mann, S. In Vitro Gene Expression within Membrane-Free Coacervate Protocells. Chemical Communications
2015, $51 \quad$ (57), 11429-11432. https://doi.org/10.1039/C5CC04220H.

(20) Drobot, B.; Iglesias-Artola, J. M.; Le Vay, K.; Mayr, V.; Kar, M.; Kreysing, M.; Mutschler, H.; Tang, T.-Y. D. Compartmentalised RNA Catalysis in Membrane-Free Coacervate Protocells. Nature Communications 2018, 9 (1), 3643. https://doi.org/10.1038/s41467-018-06072-w.

(21) Li, J.; Liu, X.; Abdelmohsen, L. K. E. A.; Williams, D. S.; Huang, X. Spatial Organization in Proteinaceous MembraneStabilized Coacervate Protocells. Small 2019, 15 (36), 1902893. https://doi.org/10.1002/smll.201902893.

(22) Poudyal, R. R.; Guth-Metzler, R. M.; Veenis, A. J.; Frankel, E. A.; Keating, C. D.; Bevilacqua, P. C. Template-Directed RNA Polymerization and Enhanced Ribozyme Catalysis inside Membraneless Compartments Formed by Coacervates. Nature Communications 2019, $10 \quad$ (1), 490. https://doi.org/10.1038/s41467-019-08353-4.

(23) Gobbo, P.; Tian, L.; Pavan Kumar, B. V. V. S.; Turvey, S.; Cattelan, M.; Patil, A. J.; Carraro, M.; Bonchio, M.; Mann, S. Catalytic Processing in Ruthenium-Based Polyoxometalate Coacervate Protocells. Nature Communications 2020, 11 (1), 41. https://doi.org/10.1038/s41467-019-13759-1.

(24) Nakashima, K. K.; van Haren, M. H. I.; André, A. A. M.; Robu, I.; Spruijt, E. Active Coacervate Droplets Are Protocells That Grow and Resist Ostwald Ripening. Nature Communications 2021, $12 \quad$ (1), 3819. https://doi.org/10.1038/s41467-021-24111-x.

(25) Gucht, J. van der; Spruijt, E.; Lemmers, M.; Cohen Stuart, M. A. Polyelectrolyte Complexes: Bulk Phases and Colloidal Systems. Journal of Colloid and Interface Science 2011, 361 (2), 407-422. https://doi.org/10.1016/j.jcis.2011.05.080.

(26) Blocher McTigue, W. C.; Perry, S. L. Protein Encapsulation Using Complex Coacervates: What Nature Has to Teach Us. Small 2020, $16 \quad$ (27), 1907671. https://doi.org/10.1002/smll.201907671.

(27) Wang, X.; Zhang, P.; Tian, L. Spatiotemporal Organization of Coacervate Microdroplets. Current Opinion in Colloid and Interface Science 2021, 52, 101420. https://doi.org/10.1016/j.cocis.2021.101420.

(28) Mu, W.; Ji, Z.; Zhou, M.; Wu, J.; Lin, Y.; Qiao, Y. MembraneConfined Liquid-Liquid Phase Separation toward Artificial Organelles. Science Advances 2021, 7 (22), eabf9000. https://doi.org/10.1126/sciadv.abf9000.

(29) Harada, A.; Kataoka, K.; Kataoka, A.; Harada, A.; Kataoka, K. Chain Length Recognition: Core-Shell Supramolecular Assembly from Oppositely Charged Block Copolymers. Science 1999, 283 (5398), 65-67. https://doi.org/10.1126/science.283.5398.65.

(30) Voets, I. K.; de Keizer, A.; Cohen Stuart, M. A. Complex Coacervate Core Micelles. Advances in Colloid and Interface Sci$\begin{array}{llll}\text { ence 2009, } 147-148 & \text { (C), 300-318. }\end{array}$ https://doi.org/10.1016/j.cis.2008.09.012.

(31) Cabral, H.; Miyata, K.; Osada, K.; Kataoka, K. Block Copolymer Micelles in Nanomedicine Applications. Chemical Reviews 2018, 118 (14), 6844-6892. https://doi.org/10.1021/acs.chemrev.8b00199.

(32) Gao; Holkar; Srivastava. Protein-Polyelectrolyte Complexes and Micellar Assemblies. Polymers 2019, 11 (7), 1097. https://doi.org/10.3390/polym11071097.

(33) Chen, F.; Stenzel, M. H. Polyion Complex Micelles for Protein Delivery. Australian Journal of Chemistry 2018, 71 (10), 768. https://doi.org/10.1071/ch18219. 
(34)

Li, J.; Kataoka, K. Chemo-Physical Strategies to Advance the in Vivo Functionality of Targeted Nanomedicine: The Next Generation. Journal of the American Chemical Society 2021, 143 (2), 538-559. https://doi.org/10.1021/jacs.0c09029.

(35) Tang, T.-Y. D.; Hak, C. R. C.; Thompson, A. J.; Kuimova, M. K.; Williams, D. S.; Perriman, A. W.; Mann, S. Fatty Acid Membrane Assembly on Coacervate Microdroplets as a Step towards a Hybrid Protocell Model. Nature Chemistry 2014, 6 (6), 527-533.

(36) Folkmann, A. W.; Putnam, A.; Lee, C. F.; Seydoux, G. Regulation of Biomolecular Condensates by Interfacial Protein Clusters. Science 2021, 373 (6560), 1218-1224. https://doi.org/10.1126/science.abg7071.

(37) Mason, A. F.; Buddingh, B. C.; Williams, D. S.; Van Hest, J. C. M. Hierarchical Self-Assembly of a Copolymer-Stabilized Coacervate Protocell. Journal of the American Chemical Society 2017, 139 (48), 17309-17312. https://doi.org/10.1021/jacs.7b10846.

(38) Mason, A. F.; Yewdall, N. A.; Welzen, P. L. W.; Shao, J.; Stevendaal, M. Van; Hest, J. C. M. V.; Williams, D. S.; Abdelmohsen, L. K. E. A. Mimicking Cellular Compartmentalization in a Hierarchical Protocell through Spontaneous Spatial Organization. ACS Central Science 2019, 5 (8), 1360-1365. https://doi.org/10.1021/acscentsci.9b00345.

(39) Yewdall, N. A.; Buddingh, B. C.; Altenburg, W. J.; Timmermans, S. B. P. E.; Vervoort, D. F. M.; Abdelmohsen, L. K. E. A.; Mason, A. F.; Hest, J. C. M. Physicochemical Characterization of Polymer-Stabilized Coacervate Protocells. ChemBioChem 2019, 20 (20), 2643-2652. https://doi.org/10.1002/cbic.201900195.

(40) van Stevendaal, M. H. M. E.; Vasiukas, L.; Yewdall, N. A.; Mason, A. F.; van Hest, J. C. M. Engineering of Biocompatible Coacervate-Based Synthetic Cells. ACS Applied Materials and Interfaces 2021, 13 (7), 7879-7889. https://doi.org/10.1021/acsami.0c19052.

(41) Mason, A. F.; Altenburg, W. J.; Song, S.; van Stevendaal, M.; van Hest, J. C. M. Terpolymer-Stabilized Complex Coacervates: A Robust and Versatile Synthetic Cell Platform. In Methods in Enzymology; Elsevier Inc., 2021; Vol. 646, pp 5182. https://doi.org/10.1016/bs.mie.2020.06.008.

(42) Zhang, Y.; Chen, Y.; Yang, X.; He, X.; Li, M.; Liu, S.; Wang, K.; Liu, J.; Mann, S. Giant Coacervate Vesicles as an Integrated Approach to Cytomimetic Modeling. Journal of the American Chemical Society 2021, 143 (7), 2866-2874. https://doi.org/10.1021/jacs.0c12494.

(43) Zhao, C.; Li, J.; Wang, S.; Xu, Z.; Wang, X.; Liu, X.; Wang, L.; Huang, X. Membranization of Coacervates into Artificial Phagocytes with Predation toward Bacteria. ACS Nano 2021, $15 \quad$ (6), 10048-10057. https://doi.org/10.1021/acsnano.1c01694.

(44) Pir Cakmak, F.; Marianelli, A. M.; Keating, C. D. Phospholipid Membrane Formation Templated by Coacervate Droplets. Langmuir 2021, 37 (34), 10366-10375. https://doi.org/10.1021/acs.langmuir.1c01562.

(45) Liu, S.; Zhang, Y.; Li, M.; Xiong, L.; Zhang, Z.; Yang, X.; He, X.; Wang, K.; Liu, J.; Mann, S. Enzyme-Mediated Nitric Oxide Production in Vasoactive Erythrocyte Membrane-Enclosed Coacervate Protocells. Nature Chemistry 2020, 12 (12), 1165-1173. https://doi.org/10.1038/s41557-02000585-y.

(46) Pir Cakmak, F.; Grigas, A. T.; Keating, C. D. Lipid VesicleCoated Complex Coacervates. Langmuir 2019, 35 (24), 7830-7840. https://doi.org/10.1021/acs.langmuir.9b00213.
Ke, C. Y.; Wu, Y. T.; Tseng, W. L. Fluorescein-5-Isothiocyanate-Conjugated Protein-Directed Synthesis of Gold Nanoclusters for Fluorescent Ratiometric Sensing of an Enzyme-Substrate System. Biosensors and Bioelectronics 2015, 69, 46-53. https://doi.org/10.1016/j.bios.2015.02.002.

(48) Spruijt, E.; Sprakel, J.; Cohen Stuart, M. A.; van der Gucht, J. Interfacial Tension between a Complex Coacervate Phase and Its Coexisting Aqueous Phase. Soft Matter 2010, 6 (1), 172178. https://doi.org/10.1039/B911541B.

(49) Qin, J.; Priftis, D.; Farina, R.; Perry, S. L.; Leon, L.; Whitmer, J.; Hoffmann, K.; Tirrell, M.; De Pablo, J. J. Interfacial Tension of Polyelectrolyte Complex Coacervate Phases. ACS Macro Letters 2014, 3 (6), 565-568. https://doi.org/10.1021/mz500190w.

(50) Li, L.; Srivastava, S.; Andreev, M.; Marciel, A. B.; De Pablo, J. J.; Tirrell, M. V. Phase Behavior and Salt Partitioning in Polyelectrolyte Complex Coacervates. Macromolecules 2018, 51 (8), 2988-2995. https://doi.org/10.1021/acs.macromol.8b00238.

(51) Lindhoud, S.; Norde, W.; Stuart, M. A. C.; Cohen Stuart, M. A. Effects of Polyelectrolyte Complex Micelles and Their Components on the Enzymatic Activity of Lipase. Langmuir 2010, $26 \quad$ (12), 9802-9808. https://doi.org/10.1021/la1000705.

(52) Bourganis, V.; Karamanidou, T.; Kammona, O.; Kiparissides, C. Polyelectrolyte Complexes as Prospective Carriers for the Oral Delivery of Protein Therapeutics. European Journal of Pharmaceutics and Biopharmaceutics 2017, 111, 44-60. https://doi.org/10.1016/j.ejpb.2016.11.005.

(53) Panova, I. G.; Khaydapova, D. D.; Ilyasov, L. O.; Umarova, A. B.; Yaroslavov, A. A. Polyelectrolyte Complexes Based on Natural Macromolecules for Chemical Sand/Soil Stabilization. Colloids and Surfaces A: Physicochemical and Engineering Aspects 2020, 590 (November 2019), 124504. https://doi.org/10.1016/j.colsurfa.2020.124504.

(54) Panova, I. G.; Ilyasov, L. O.; Khaidapova, D. D.; Bashina, A. S.; Smagin, A. V.; Ogawa, K.; Adachi, Y.; Yaroslavov, A. A. Soil Conditioners Based on Anionic Polymer and Anionic Micro-Sized Hydrogel: A Comparative Study. Colloids and Surfaces A: Physicochemical and Engineering Aspects 2021, 610 (June 2020), 125635. https://doi.org/10.1016/j.colsurfa.2020.125635.

(55) Liu, B.; Fan, Y.; Li, H.; Zhao, W.; Luo, S.; Wang, H.; Guan, B.; Li, Q.; Yue, J.; Dong, Z.; Wang, Y.; Jiang, L. Control the Entire Journey of Pesticide Application on Superhydrophobic Plant Surface by Dynamic Covalent Trimeric Surfactant Coacervation. Advanced Functional Materials 2021, 31 (5), 2006606. https://doi.org/10.1002/adfm.202006606.

(56) Huang, B.; Chen, F.; Shen, Y.; Qian, K.; Wang, Y.; Sun, C.; Zhao, X.; Cui, B.; Gao, F.; Zeng, Z.; Cui, H. Advances in Targeted Pesticides with Environmentally Responsive Controlled Release by Nanotechnology. Nanomaterials 2018, 8 (2), 102. https://doi.org/10.3390/nano8020102.

Schmitt, C.; Turgeon, S. L. Protein/Polysaccharide Complexes and Coacervates in Food Systems. Advances in Colloid and Interface Science 2011, 167 (1-2), 63-70. https://doi.org/10.1016/j.cis.2010.10.001.

(58) Martins, I. M.; Barreiro, M. F.; Coelho, M.; Rodrigues, A. E. Microencapsulation of Essential Oils with Biodegradable Polymeric Carriers for Cosmetic Applications. Chemical Engineering Journal 2014, 245, 191-200. https://doi.org/10.1016/j.cej.2014.02.024. 
Table of Contents artwork

Stable Complex Coacervate Emulsions

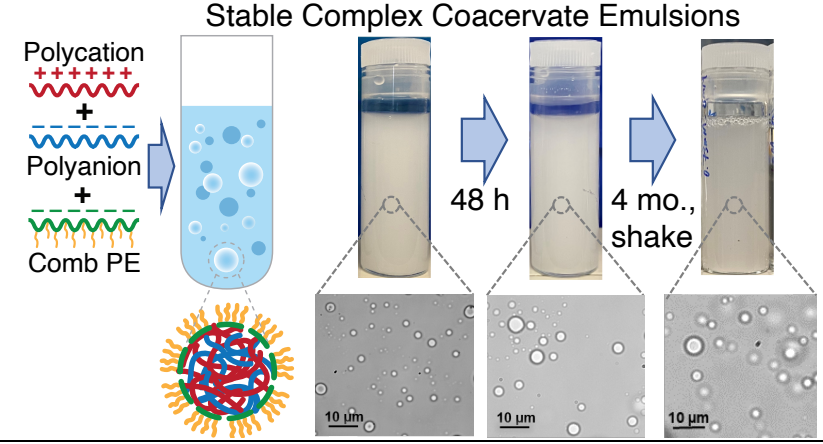

